\title{
"ARRE NOBLASTOMA"
}

\author{
Dr. Armando Moreno G. \\ Prof. Rubén Darío Guzmán \\ Prof. Alvaro Fonnegra $M$. \\ Prof. Ricardo Alvarado Pantoja
}

El conocimiento de las características clínicas, la incidencia y comportamiento de este raro tumor ovárico, en nuestro medio está aún lejos de alcanzarse por la carencia de un sistema nacional de información y registro de tumores ováricos; motivación del médico para envío de las piezas quirúrgicas, para estudio anatomopatológico y carencia de recursos adecuados para envío, estudio y clasificación de las muestras, así como seguimiento de los casos.

En este pequeño trabajo informamos de cuatro (4) casos de Arrenoblastoma, manejados en nuestro Servicio en los últimos $15 \frac{1}{2}$ años, constituyendo con estos, un total de nueve (9), informados en nuestra literatura médica, que agrupados y analizados, por lo menos, sus características más importantes acerca de su comportamiento biológico en nuestra población femenina.

\section{Material y Métodos}

Se revisaron los protocolos quirúrgicos y de autopsia de nuestro Departamento de Patología desde el 10 de Enero de 1960 hasta el 30 de Junio de 1975, seleccionándose los casos de Arrenoblastoma, analizándose las historias clínicas correspondientes, así como los cortes histológicos archivados de cada uno de los mismos.

Se consultó la literatura colombiana acerca de este tumor, estudiándose los casos publicados y revisándose los cortes microscópicos coleccionados de todos ellos menos de 1, del Servicio de Ginecología del Hospital de San Vicente, de Medellín.

Finalmente, con el ánimo de conocer el comportamiento de este tumor en forma más aproximada, en nuestro medio, se analizaron los aspectos más importantes de los casos publicados y los 4 encontrados en los $15 \frac{1}{2}$ años que abarca la presente revisión y se comparan con lo consignado en la literatura extranjera.

\section{Resultados}

Durante el período que comprende este estudio hubo 440.360 hospitalizaciones de las cuales 1.210 correspondieron a tumores de los ovarios: esto es el $0.25 \%$.

En este grupo de 1.210 tumores ováricos hubo 16 tumores del estroma gonadal ( 7 de la granulosa; 2, tecagranulosa; 3, tecomas; 4, arrenoblastomas), o sea, el $1.25 \%$ de todos los tumores ováricos. (Ver tumores funcionales de esta revisión). Los 4 
arrenoblastomas constituyeron el $25 \%$ de los tumores funcionales y el $0.25 \%$ de todos los tumores ováricos.

La edad promedio fue los 21 años.

\section{El cuadro clínico:}

Esencialmente estuvo constituído por oligomenorreas, seguidas de amenorrea secundaria, excepto 1 paciente de 17 años con amenorrea primaria. Todas presentaron signos francos de virilización (voz grave, hirsutismo, hipertrofia del clítoris, hipotrofia mamaria, contorno corporal masculino, etc.).

Al examen, presencia de masa ovárica.

\section{Antecedentes:}

En 3 casos la menarquia fue normal.

2 presentaron ciclos normales por espacio de varios años.

1 oligomenorreas, antes de instalarse la amenorrea.

5a 4: paciente, ya dijimos, presentaba amenorrea primaria.

\section{La Paridad:}

Tres (3) pacientes tenían vida sexual activa por espacio de 3-6 años, una de las cuales presentaba esterilidad primaria.

Las otras dos (2): una, un embarazo con parto normal $2 \frac{1}{2}$ años antes de instalarse la amenorrea y esterilidad secundaria.

La otra, cuatro (4) embarazos y partos normales, el último, 18 meses antes de su ingreso.

\section{Labcratorio y Rayos $\mathrm{X}$ :}

Cuadro Hemático normal en 3 pacientes. Una presentó Hematocrito y
Hemoglobina aumentados (51 y 16,5 g.). les.

Examen parcial de orina: Norma-

Química Sanguínea: normales.

En Orina de 24 horas: 17\%: elevados en un caso, 28,2 mg.

17-OH: normales en todas.

Los estudios radiológicos de Tórax, Abdomen simple y Silla turca, fueron normales en todos los casos.

Citología Vaginal funcional: en todos fue informado como frotis atrófico, (100-0-0).

En dos (2) se intentó biopsia de endometrio sin obtenerse material suficiente para estudio de Anatomía Patológica.

El avario afectado fue el izquierdo en tres (3) y el derecho en uno (1). Todos fueron unilaterales.

Tamaño: el menor de $8 \mathrm{cms}$. de diámetro, el mayor de $28 \mathrm{cms}$.

Promedio: $19 \mathrm{cms}$.

\section{Métode clínico quirúrgico:}

A la Laparotomía, 2 casos presentaban adherencias locales; 1 caso presentaba ascitis, y el 4 \% caso, tumor limitado de ovario con cápsula intacta.

\section{Microscopía :}

Tres (3) casos eran de la variedad intermedia; un (1) caso francamente indiferenciado.

\section{Tratamiento:}

En todos los cuatro (4) casos se practicó S.O. Unilateral en primera instancia. En dos (2) casos se complementó con H. + S.O. Contralateral y Omentectomía; 1, dos meses 
después con diagnóstico de "Arrenoblastoma maligno". El otro, a los 5 meses cuando en un control se palpó masa en el sitio de la S.O.

A la reexploración quirúrgica se encontró tejido tumoral entre las hojas del ligamento ancho, ligamento infundíbulo-pélvico y retroperitonealmente tumor sobre los vasos ilíacos, lo que se interpretó como crecimiento tumoral a partir de tejido dejado en la sección del pedículo ovárico en la primera intervención.

\section{Supervivencia :}

Una paciente falleció a los 9 meses; pjresentaba adherencias en el sitio de la masa tumoral, variedad histológica intermedia.

Un caso, reproducción tumoral en el sitio original a los 5 meses. Esta paciente presentaba líquido ascítico, el cual no fue estudiado para células tumorales.

Los otros dos casos sobreviven en buenas condiciones. Uno por 3 años, $y$ el otro 5 meses.

\section{Involución de los signos y síntomas:}

Excepto la paciente que falleció a los 9 meses en carcinomatosis, las otras 3 pacientes presentaron menstruación entre los 2 y 3 meses, los signos de virilización involucionaron parcialmente.

\section{Comentarios:}

Sinónimos: Androblastoma, Tumor de Sertoli Leydig.

\section{I - Historia}

Pick en 1905 fue el primero en describirlo denominándolo "Adenoma Tubulare testiculare ovarü. Meyer originó el término arrenoblastoma, puntualizando que puede originar virilización y que característicamente con- tiene "formaciones tubulares que recuerdan los condones sexuales". Más tarde el mismo Meyer lo clasificó en 3 tipos histológicos (13).

Scully, entre otros, lo denominó Tumor de Sertoli-Leydig. En nuestro país, los primeros casos se publican en el año de 1950.

\section{II - Frecuencia}

Es el tumor virilizante más frecuente del ovario; no obstante representa menos del $1 \%$ de los tumores ovári$\cos$ y es $5-10$ veces menos frecuente que los tumores de la teca-granulosa. En Colombia no pasan de 6 los casos publicados desconociéndose su verdadera incidencia. En este Centro Hospitalario hemos visto y manejado 4 casos en los últimos 15 años (Cuadro № 1), uno de los cuales nos fue remitido después de su extirpación pero actualmente se encuentra en control en el I.M.I., constituyendo el $0.24 \%$ de los 1.210 tumores ováricos estudiados en este lapso.

\section{III - Edad del diagnóstico}

Se presenta con mayor frecuencia entre los 20 y 40 años. La edad promedio ha sido señalada por varios autores en los 34 años $(13,10)$. En los casos publicados en Colombia y en los 4 nuestros de esta serie, la edad promedio es de 22 años, excluyendo un caso publicado por Meleg, Suzanne (9) de 5 años de edad (Cuadro № 1).

Aun cuando raro antes de la pubertad, han sido informados varios casos en niñas, Novak (11) cita una niña de 30 meses, Norris (10) una de 4 años, Melag (9) una de 5 años. Así mismo son varios los casos reportados en mujeres ancianas; O'Hern (13) cita una paciente de 78 años. Fero en general el $75 \%$ de las pacientes son menores de 40 años $(11,12)$. 


\title{
CUADRO № 1
}

\author{
- Total de Tumores Ováricos: \\ - Frecuencia: \\ - Total pacientes ginecoobstétricas:

\section{IV - Cuadro Clínico}

Se puede resumir en 2 fases: I de desfeminización. II de virilización. Los primeros incluyen: oligomenorreas, amenorrea, $(100 \%$ en nuestros casos) atrofia mamaria, desaparición de los depósitos de grasa subcutánea que dan redondez a la figura femenina, esterilidad los segundos, hirsutismo, carácter grave de la voz, e hipertrofia del clítoris, todos presentes en nuestras pacientes.

Sin embargo, debe tenerse en cuenta que el cuadro clínico varía según el tiempo de evolución transcurrido al momento del examen. No es raro que en niñas se acompañe de pubertad precoz, como se ve en casos informados $(13,9)$ e incluso sin manifestaciones clínicas (13) o que sea hallado accidentalmente en intervención abdominal para otro fin o en autopsia (13) cerca del $15 \%$ de los estudiados por Novak (12) resultaron inertes.

Aunque infrecuente han sido reportados 10 casos, por lo menos, coexistentes con embarazo, en nuestro medio ninguno ha coexistido con embarazo.

Se ha informado de varios casos de familias en los cuales varios miembros han sido afectados de arrenoblastoma. Goldstein y Colb (16) publicaron en 1970, 2 casos en 2 primas de primer grado; una de 17 años y otra de 15 años, cromosómicamente normales. Norris (10) una paciente de 4 años de edad, perteneciente a una familia con otros miembros afectos de la misma neoplasia. No ha sido informado en nuestra literatura médica antecedentes hereditarios. Ha sido destacada la asociación de arrenoblastoma con anomalías tiroideas (10). Este hecho no se ha confirmado en nuestros casos.

\section{$V$ - Diagnóstico diferencial}

El arrenoblastoma, debe diferenciarse, en su cuadro clínico, de todas aquellas entidades que presenten virilización. Especialmente, la hiperplasia adrenal, sindrome de Cushing, anormalidades genéticas (10), luteoma del embarazo, que en ocasiones cursa con virilización, el hirsutismo idiopático, adenocarcinoma metastásico y primario del ovario, que en ocasiones cursa con luteinización del estroma y efectos esteroidógenos androgénicos; el tumor de células lípidas del ovario, etc.

\section{VI - Laboratorio}

Los 17 atosteroides se elevan en $1 / 3$ parte de las pacientes virilizadas $(10,12)$. En los casos publicados en nuestro medio a los cuales se les ha practicado este examen no ha habido aumento por encima de los valores normales.

En uno de nuestros cuatro (4) casos hubo un aumento moderado de los $17 \mathrm{~K}$, que descendió tras la extirpación del tumor.

Parece que el arrenoblastoma produce grandes cantidades de testosterona, y puesto que ésta contribuye muy poco a los niveles urinarios de 
$17 K$, estos se encuentran casi siempre normales o muy ligeramente aumentados en estas pacientes $(5,11$, 16).

Se ha confirmado que el arrenoblastoma produce testosterona, androstenendiona y Dihidroepiandrosterona.

In vitro, l el tejido del arrenoblastoma es aproximadamente 13 veces más efectivo en la conversión de androstenendiona $y$ dihidroepiandrosterona a testosterona (4). En análisis directo del tejido del tumor y de la sangre venosa procedente del mismo, se ha confirmado la producción de andrógenos y en algunos casos se ha podido identificar una vía biosintética de deshidrogenosa delta 3,5 betaob que soslaya la progesterona con incremento en la formación de andrógenos y menores cantidades de estrógenos (10).

Las pruebas de supresión con Dexametasona, parece que adquieren en el presente alguna importancia en estos pacientes en cuanto a diagnóstico diferencial se refiere, combinándolos con cuantificación de 17-K, testosterona y corticoides y estimulación con H.C.G., A.C.T.H. y depresión.

\section{VALORES HORMONALES EN EL DIAGNOSTICO DIFERENCIAL DEL ARRENOBLASTOMA CON ESTROGENOS O PROGESTERONA}

\begin{tabular}{|c|c|c|c|c|}
\hline Valores previos & $\begin{array}{c}\text { Test } \\
\text { de H.C.G. }\end{array}$ & $\begin{array}{c}\text { Estímulo } \\
\text { Adrenal ACTH }\end{array}$ & $\begin{array}{l}\text { Depresión } \\
\text { ovárica } \\
\text { con Estróg. }\end{array}$ & Dexametas. \\
\hline 17 Ks-Ligeran. S. & $17 \mathrm{k}-+$ & $17 \mathrm{k}-++$ & $17 \mathrm{~K} * * *$ & $17 \mathrm{~K} *$ \\
\hline $\begin{array}{l}\text { Testosterona } \\
\text { moderadamente } \mathrm{S} \text {. }\end{array}$ & $T-++$ & $\mathrm{T}-\mathrm{O}$ & $\mathrm{T}-* \star *$ & $\mathrm{~T}-\mathrm{O}$ \\
\hline $\begin{array}{l}\text { Corticosteroides } \\
\text { — normales }\end{array}$ & $c-O$ & $c-+$ & $c-0$ & $c-*$ \\
\hline
\end{tabular}

O: ningún cambio

+ : aumento

*: disminución

En algunos de estos pacientes existe la posibilidad de eritropoyesis estimulada por andrógenos (10).

\section{VII - Histogénesis}

En el presente aún se discute acerca de la génesis de estos tumores y de su actividad andrógena.

Se ha sugerido su origen teratomatoso y que ocurre de generación en el sentido de elementos testiculares andrógenos. También se ha propuesto que las células virilizantes derivan del hilio ovárico (15).
Según Cohnhein, derivan de restos celulares persistentes que pueden más tarde ser el núcleo de algunas neoplasias (11).

Meyer ha propuesto que en los estadios más avanzados de la diferenciación masculina pueden quedar en la porción modular del ovario originando posteriormente cuadros masculinizantes (11) entre estos el del arrenoblastoma.

Actualmente se sostiene su origen, a partir de células del estroma gona- 
dal especializado, esto es, con potencial funcional, y este concepto implica que las células especializadas del estroma ovárico pueden dar origen a tecomas, tumores de la granulosa, arrenoblastomas, etc.

Jenson y Feehner (6) en 1969, en un caso estudiado con microscopía electrónica encontraron que las células de Sertoli del tumor eran similares a las células de la granulosa, y menos parecidas a las de Sertoli del testículo. Las células de Leyding, aun cuando similares a las del testículo no mostraron cristales de Reinke y presentaban cromatina nuclear femenina, este mismo hecho ha sido destacado por O'Hern y Newbacker. Además, encontraron ocasionalmente células aparentemente de transición mostrando aspecto, tanto de células de Sertoli como de Leyding. Ellos encontraron que las células de Leyding mostraban lípidos intramitocondriales, es decir, aparentemente se mostraban responsables de la producción de hormonas; esto mismo lo habían descrito antes Dubronsky y Stou en 1965 (6, 13).

\section{VIII - Anatomía patológica}

Al tiempo de la operación estos tumores por lo general son de tamaño mediano. En su serie de 31 casos O'Hern y Newbacker, encontraron un tamaño promedio de $9.7 \mathrm{cms}$. con extremos de 2,5 y $2,4 \mathrm{cms}$. Novak (11) cita un caso de $12 \mathrm{kgr}$. de peso, y Pedawetz, señala algunas de más de $25 \mathrm{cms}$. (6).

En los casos publicados en Colombia existe uno de $40 \times 25 \mathrm{cms}$. (3) Echeverry, y otro de $27 \mathrm{cms}$. y 4.600 gr. (1) Castro Márquez. En esta revisión informamos uno de $28 \mathrm{cms}$. y $3.100 \mathrm{gr}$. y otro de $25 \mathrm{cms}$. de diámetro y $5.300 \mathrm{gr}$. de peso.

Tamaño promedio $19 \mathrm{cms}$.
El arrenoblastoma es unilateral en el $95-96 \%$ de los casos $(10,11,12)$ OíHern y Newbecker en su revisión encontraron 20 del ovario derecho y 10 del izquierdo. En Colombia, casos publicados, 3 derechos y 6 izquierdos. Todos unilaterales. La consistencia alterna desde duros a blandos.

Sólidos a quísticos especialmente los de gran tamaño; su coloración puede ser amarilla, por el gran contenido de grasa, grises violáceos, o rojizos, y con frecuencia presentan áreas focales de hemorragia y necrosis.

\section{IX - Histología}

Por estudio microscópico se comprueba que el tumor es una neoplasia del estroma gonadal, relativamente primitivo que tiende a reproducir etapas tempranas de gonadogénesis, sobre todo de diferenciación testicular y se caracteriza por cuatro constituyentes básicos combinados en su interior, que incluyen: 1) Estructuras que recuerdan a los túbulos senuníferos inmaduros conteniendo células de tipo Sertoli; 2) Células parecidas a las de Leyding; 3 ) Areas de túbulos análogos a la red ovárica 0 epididinosa; 4) Substancia fundamental del estroma primitivo (10).

Meyer (13) fue el primero en dividir el arrenoblastoma en tres tipos histológicos: Tipo I: estructuras tubulares bien diferenciadas como elemento constituyente dominante en todo el tumor y células morfológicamente análogas a células de Settoli, a menudo sin signos clínicos de virilización. Tipo 1I: una forma intermedia presentaba túbulos similares a los túbulos testiculares en todas las etapas de gonadogénesis y apariencia variable de las formaciones tubulares dentro del mismo tumor. Tipo III: tipo sarcomatoide, con estroma pleo- 
mórfico y solamente unas pocas formaciones tubulares rudimentarias.

Los tipos II y III usualmente son virilizantes. Sin embargo los signos y síntomas clínicos de virilización no son considerados esenciales para el diāgnóstico histológico. En esta serie 6 de tipo II y 3 de tipo III todos virilizantes, excepto uno de tipo III feminizante.

El estroma primitivo puede ser sarcomatoso y a veces se diferencia en músculo estriado y cartílago habiéndose informado en varios casos epitelio mucinoso y células argentófinas.

En familias con tendencia a desarrollar arrenoblastomas son también frecuentes los cistoadenomas mucinosos multiloculares, lo cual refuerza la posibilidad de que algunos cistadenomas mucinosos multiloculares, sean expresión unilateral de un arrenoblastoma oculto (10). Histológicamente surge un problema diagnóstico en la diferenciación de este tumor con el adenocarcinoma primario y metastásico. O'Hern y Newbecker (13) citan 7 casos de adenocarcinoma mal diagnosticados como arrenoblastomas. Los carcinomas ováricos pueden inducir luteinización del estroma y mostrar un gran parecido con los arrenoblastomas ya que las células luteinizadas son parecidas a las de Leydig y sus funciones con efecto estrógeno o andrógeno. Estos mismos autores citan en su serie 3 pacientes que tenían teratoma quístico adulto; 2 en el ovario opuesto y 1 en el mismo ovario con el arrenoblastoma.

En la serie estudiada por Novak (12) 5 pacientes tenían teratoma maligno asociado. Otros 7 casos tenían cambios mucinosos. Existe un caso en la literatura de un arrenoblastoma asociado con tumor carcinoide.
Sitio de metástasis: En los casos que se presentan, las metástasis y la invasión ocurren como en cualquier tumor ovárico maligno primariamente con extensión intraabdominal $(67 \%), 0$ recurrencia y raramente metástasis distantes (12). La diseminación linfática y hematógena ocurre en menos del $20 \%$ de los casos.

\section{$X$ - Comportamiento biológico y malignidad}

Varias revisiones han sido publicadas y con alguna regularidad aparecen artículos donde se reportan 1, 2 - 3 casos. Merecen especial mención las de Joret y Fenn quienes revisaron 122 casos incluyendo 3 propios con un promedio de malignidad de $22 \%$.

Fedowitz y O. Bren, en 1960, en 240 casos reportados y agregando 2 propios determinaron una incidencia de malignidad del $21.3 \%$; sus dos casos fueron seguidos por 9 y 12 años sin evidencia de recurrencia o metástasis.

Foss y Leverton, reportaron un estudio de seguimiento de 3 casos; uno por 5 años y otros dos por 12 años permaneciendo asintomáticos y sin evidencia de recurrencia o metástasis. De estos casos, dos tratados conservativamente tuvieron un total de $4 \mathrm{em}$ barazos posteriores al tratamiento.

O'Bern y Newbecker, ya citados anteriormente en su serie de 31 casos, reportaron seguimiento en 29 casos por un promedio de 5,5 años, 8 durante 4 años, 4 por 10, 13, 22 y 25 al tiempo del estudio (1962), 25 vivas y sanas, 4 pacientes de su serie murieron, una por el tumor clasificado histológicamente como Tipo III de gran tamaño con invasión local y metástasis al momento del diagnóstico, Los otros 3 casos fallecieron sin haber presentado recurrencias ni metástasis por otra causa, dando un 
porcentaje de malignidad del $3 \%$, quizá el más bajo expresado hasta el presente en contraste con Novak que cita un $33 \%$ de recidiva; Robbin's $20 \%$; Pedowitz $21.3 \%$ Javet y Finn $22 \%$.

Es interesante conocer cual fue el tratamiento realizado a los pacientes de su serie.

A 18 pacientes les fue practicada Salpingo-ooforectomía unilateral; en 2 casos solamente Ooforectomía parcial; en 9 casos Histerectomía + S. O. B.; a una paciente de 14 años le fue practicado esto último; e irradiación después de que confundió el diagnóstico con el de teratoma maligno.

En un estudio sobre 111 casos publicados en 1965 (12) se relaciona tratamiento y mortalidad. 78 pacientes: Ooforectomía + Salpinguectomía unilateral: 13 muertos. 29 pacientes: Histerectomía + S.O.B.: 9 muertos. 4 escisión parcial: 100\% de mortalidad.

Destacan los autores que cuando hubo recurrencia en la mayoría de los casos, el anexo conservado no estaba comprometido a pesar de una carcinomatosis generalizada. No sabemos las características de los tumores al momento de la intervención.

Greenblat y Gambrell en 1972 (4) reportaron 3 casos de 26, 18 y 16 años, con tumores bien encapsulados a quienes trataron con Salpingo-ooforectomía unilateral. En esta recopilación de casos en Colombia la malignidad en términos de mortalidad - recurrencia es de $4: 9$ o sea $44 \%$. Es interesante anotar que solamente un caso de estos era de Tipo III, los otros eran Tipo 11.

\section{Tratamiento}

Parece no existir una correlación histológica de malignidad y supervivencia, resultando más significativo el aspecto macroscópico siendo este primariamente a la Laparotomía el que determina la terapia $(10,11,12$, 16) especialmente cuando se trata de mujeres en quienes son importantes los embarazos futuros.

El comportamiento clínicamente maligno de estos tumores es tan bajo que se justifica la conservación del útero y ovario opuestos en estos pacientes (10).

Sin embargo, si existe uno o más de los siguientes criterios debe practicarse cirugía radical $(13,12)$.

1 Bilateralidad

2 Ascitis

3 Adherencias tumorales

4 Excrecencias externas en la cápsula

5 Invasión local

6 Implantación peritoneal

7 Salida de células tumorales a la cavidad en el momento de la operación

8 Evidencia macroscópica o microscópica, de invasión linfática o vascular. El tamaño, solamente no es criterio de importancia pronóstica; Hartz informa un caso de 25 libras, tratamiento conservativo, vivo y bien.

En la mujer de edad no existe objeción a la práctica de la Histerectomía total y S.O.B. Si hay extensión debe pensarse en la irradiación aunque la eficacia de la radioterapia y la quimioterapia en este grupo de tumores no está muy comprobada. 


\section{XIII - Evclución de los síntomas después del tratamiento}

La regresión de los síntomas, habitualmente es incompleta en lo que respecta a la virilización. El primero es el retorno de la menstruación y generalmente los síntomas desaparecen en el mismo orden en que han aparecido.

En su serie, O'Hern y Newbecker (13), encontraron que de 16 pacientes que después de su tratamiento fueron catalogados aptos para menstruar, 9 lo hicieron entre 1 y 3 meses. En cuanto al embarazo se refiere a 13 de sus pacientes, aptos para llegar a él. Su historia gestacional fue avaluada en 5 y de estos, 3 llegaron al embarazo teniendo un total de 5 niños. Ya citamos anteriormente los 2 casos de Foss y Leverton con 4 embarazos posteriores al tratamiento.

En esta serie los casos afortunados presentaron menstruaciones entre los 2 y 3 meses después de la extirpación.

\section{BIBLIOGRAFIA}

1 CASTRO MARQUEZ J.: "Hirsutismo". Revista Colombiana de Obstetricia y Ginecología. Vol. XVI No 2, 1965. Pág. 121-146-

2 DIAZ I. RENE e ISAZA M. GUSTAVO: "Arrenoblastoma del Ovario". Antioquia Médica.

$1: 191,1950$.

3 ECHEVERRY GONZALO, BARRIOS BERNARDO: Arrenoblastoma ovárico con degeneración maligna. Revista Colombiana de Obstetricia.
4 GREENBLAT R., CANBELL R.: Arrhenoblastoma, Three Cases, Obst. Gynec. 39: 567. 1972.

5 GOLDSTEIN DAVID, EMMET LAMB: Arrhenoblastoma. first cousins, report two cases. abst. and Yinec. 35: 444, 1970.

6 JANONSKY N., PARANENON DHAN T. L. Mayor problemen Obstetries and Yinecology. Vol. 4, Pág. 72-78, Philadelphia, 1973.

7 LOPEZ ESCOBAR J. Arrenoblastoma ovárico Med. y Cirugía. 14: 199, 1950.

8 LOPEZ ESCOBAR J.: Tumores Displásicos del ovario, Arrenoblastoma. 15: 401, 1951.

9 MELEG YUSANNE: Arrenoblastoma Feminizante Prepublal. Tribuna Médica.

10 M. MORRIS HENRY; CHARLTON TOM: TUmores funcionales del Ovario. Clínicas obstétricas y ginecológicas. Pág. 195-199. Vol. $1,1975$.

11 NOVAK EDMUND: Tratado de Ginecología. Arrenoblastoma; VIII Ed. Mex. 1972. Pág. 524-35.

12 NOVAK EDMUND; LONG HERMAN: Arrenoblastoma of the ovary, a review of the Ovarium tumor rgeistry. Am. Journal of Obst. and Ginec. Vol. 92: 1.082, 1965.

13 O'HERN THOMAS; NEWBECKER ROBERT: Arrenoblastoma. Obst. and Gynec. 19758 , 1960.

14 RADMON MELVIN; ITRUMNER DELI; BHAGAVAN BELEUR: Arrenoblastoma of the ovary (presentation of on case report a discusion of the diferential diagnosis). Am. J. of Obstetrics anl Gynecology. 106: 1187. 1970.

15 ROBLIN'S STANLEY: Tratado de patología. IV Ed. Editorial Interamericana S. A. México 197 pág.

16 YOUNGLY EDWARD; RICHMOND HUGH; ATYCO RALPH: Arrenoblastoma in vivo and in vitro. Studies am. J. of Obstetrics and Gynecology. 116: 401 1973. 
CUADRO № 2

\begin{tabular}{lcccc}
\hline Autor & No & Año & Edad & Edad promedio \\
\hline López Escobar & 1 & 1950 & 20 & \\
Díaz e Isaza & 2 & 1950 & 18 & 22 años \\
Echeverry y Barrios & 3 & 1955 & 23 & \\
Castro M. & 4 & 1959 & 28 & (Excluyendo la paciente \\
Melag, Suzanne & 5 & 1968 & 5 & de 5 años) \\
I. M. I. & 6 & 1969 & 22 & Literatura extranjera: \\
H. C. No 529894 & 7 & 1972 & 22 & E: 34 azos \\
& 8 & 1975 & 22 & \\
\hline 713072 & 9 & 1975 & 17 & \\
\hline
\end{tabular}

CUADRO № 3

CUADRO CLINICO

\begin{tabular}{|c|c|c|c|c|c|c|c|c|}
\hline $\mathbf{N}^{0}$ & Edad & Menarquia & Paridad & Ciclos anteriores & Trastorno del ciclo & Voz grave & Hirsutismo & Clítoris \\
\hline 9 & 20 & 14 años & Est. Primaria & $15-30 \times 8 \mathrm{~d}$ & Amenorrea 2 años & + & + & + \\
\hline 2 & 18 & 13 años & Est. Primaria & $30 \times 3$ & Amenorrea 3 años & + & + & + \\
\hline 3 & 23 & 14 años & Est. Primaria & $28 \times 3$ & Amenorrea 2 años & + & + & - \\
\hline 4 & 28 & 16 años & Est. Primaria & $30 \times 3$ & Amenorrea $2 \frac{1}{2}$ años & + & + & + \\
\hline 5 & 5 & Impub. & - & - & Pub. precoz & - & - & - \\
\hline 6 & 22 & 15 años & Est. Primaria & $28 \times 3 \times 4$ & Amenorrea 4 años & + & + & + \\
\hline 7 & 22 & 14 años & G1 P1 & $20-40 \times 3-5$ & Amenorrea $1 \frac{1}{2}$ años & - & + & + \\
\hline 8 & 24 & 12 años & G4 P4 A0 & $28 \times 30 \times 8-5$ & Amenorrea $1 \frac{1}{2}$ años & + & + & + \\
\hline 9 & 17 & A. Primar. & - & - & Amenorrea Primaria & + & + & + \\
\hline 9 & 22 & & $5 / 9$ & & $100 \%$ & $7 / 9$ & $8 / 9$ & $7 / 9$ \\
\hline
\end{tabular}


CUADRO N: 4

\begin{tabular}{|c|c|c|c|c|c|c|c|}
\hline Casos & $\begin{array}{l}\text { Ovario } \\
\text { afectado }\end{array}$ & Tamaño & Peso & $\begin{array}{l}\text { Características } \\
\text { a la Laparotomía }\end{array}$ & Tratamiento & Grado & Supervivencia \\
\hline 1 & Derecho & $8 \mathrm{cms}$. & - & Ltado. al ovario & S. O. D. & II & Mr 10 años \\
\hline 2 & Izquierdo & $12 \mathrm{cms}$. & $280 \mathrm{~g}$. & Ltado. al ovario & S. o. 1. & 11 & Mr 2 años \\
\hline 3 & Izquierdo & $25 \mathrm{cms}$. & $1.700 \mathrm{~g}$ & Adherencias & S. O. I. & 11 & $21 / 2$ años + \\
\hline 4 & Izquierdo & 27 cms. & $4.600 \mathrm{~g}$ & Ltado. al ovario & S. O. I. & 111 & Mr 2 años \\
\hline 5 & Derecho & $20 \mathrm{cms}$. & $647 \mathrm{~g}$ & Adherencias + ascitis & H. + S. O. B. & 111 & $2 \frac{1}{2}$ meses + \\
\hline 6 & Izquierdo & $28 \mathrm{cms}$. & $3.100 \mathrm{~g}$ & Adherencias & S. O. I. & II & 9 meses + \\
\hline 7 & Izquierdo & $25 \mathrm{cms}$. & $5.300 \mathrm{~g}$ & Adherencias & S. O. I. & 111 & Mr 3 años \\
\hline 8 & Derecho & $8 \mathrm{cms}$. & $280 \mathrm{~g}$ & Ltado. al ovario & S. O. D. & II & $\begin{array}{l}\text { Mr } 5 \text { meses } \\
\text { Reproducción }\end{array}$ \\
\hline 9 & $\begin{array}{l}\text { Izquierdo } \\
\text { I: } 6 \\
\text { D: } 3\end{array}$ & $15 \mathrm{cms}$ & $3.000 \mathrm{~g}$. & Ascitis & S. O. 1. & $\begin{array}{lll}11 & \\
6 & T . & 11 \\
3 & T . & 111\end{array}$ & 5 meses \\
\hline
\end{tabular}

\section{CUADRO N: 5}

CONDUCTA

1 - Siempre Quirúrgica

- Apreciación cuidadosa del campo quirúrgico

- Si existe líquido ascítico: Tomar muestra para estudio citológico

- Bxp or congelación especialmente de sitio de adherencias o posible invasión

$2-\mathrm{H}+$ S.O.B. si :

- Paciente mayor de 40 años

- Si no existe deseo de maternidad

- Si líquido ascítico

- Si adherencias

- Si invasión local

- Si nódulos en la cápsula

- Si implantación en otros órganos

- Si ruptura accidental de la cápsula

- Si bilateralidad

3 - En caso contrario S.O.U. lo más lejos posible al ovario. 